\title{
Modelling of pulsed and steady-state DEMO scenarios
}

G. Giruzzi ${ }^{1}$, J.F. Artaud ${ }^{1}$, M. Baruzzo ${ }^{2,4}$, T. Bolzonella ${ }^{2}$, E. Fable ${ }^{3}$, L. Garzotti ${ }^{4}$,

I. Ivanova-Stanik ${ }^{5}$, R. Kemp ${ }^{4}$, D.B. King ${ }^{4}$, M. Schneider ${ }^{1}$, R. Stankiewicz ${ }^{5}$, W. Stępniewski ${ }^{5}$, P. Vincenzi², D. Ward ${ }^{4}$, R. Zagórski ${ }^{5}$

${ }^{1}$ CEA, IRFM, F-13108 Saint-Paul-lez-Durance, France.

${ }^{2}$ Consorzio RFX-Associazione EURATOM ENEA per la Fusione, I-35127 Padova, Italy

${ }^{3}$ Max-Planck-Institut für Plasmaphysik, EURATOM Association, 85748 Garching, Germany

${ }^{4}$ CCFE, Culham Science Centre, Abingdon OX14 3DB, UK

${ }^{5}$ Institute of Plasma Physics and Laser Microfusion, EURATOM Association, 01-497 Warsaw, Poland

\begin{abstract}
.
Scenario modelling for the demonstration fusion reactor (DEMO) has been carried out using a variety of simulation codes. Two DEMO concepts have been analysed: a pulsed tokamak, characterised by rather conventional physics and technology assumptions (DEMO1) and a steady-state tokamak, with moderately advanced physics and technology assumptions (DEMO2). Sensitivity to impurity concentrations, radiation, heat transport models has been investigated. For DEMO2, the impact of current driven non-inductively by Neutral Beams has been studied by full MonteCarlo simulations of the fast ion distribution. The results obtained are a part of a more extensive R\&D effort carried out in the EU in order to develop a viable option for a DEMO reactor, to be adopted after ITER for fusion energy research.
\end{abstract}




\section{Introduction}

The construction of a demonstration fusion reaction (DEMO) is considered an essential step for the development of fusion energy, between ITER and the first prototype of a commercial reactor. However, various DEMO concepts are being considered by the ITER partners and all of them are now working at concept selections, involving both technology and physics assessments. In particular, a vigorous programme is now being carried out in the EU, aiming at a more and more refined selection of the DEMO design. The general strategy adopted consists in developing two DEMO concepts in parallel: a pulsed tokamak, characterised by rather conventional physics and technology assumptions (DEMO1) and a steady-state tokamak, with moderately advanced physics and technology assumptions (DEMO2) [1]. The physics assessment part of this programme involves three main steps: i) the analysis of the general physics guidelines of a tokamak DEMO [2]; ii) the search for optimum working points, performed by means of systems codes, i.e., 0-D codes combining both physics and technology constraints [3]; iii) space and time dependent simulations of plasma scenarios, performed by means of integrated modelling codes with various levels of assumptions. In this last area of work, a coordinated effort has been undertaken at the EU level [4], with the general goal of analysing the working points produced by the systems code PROCESS [3] for both DEMO1 and DEMO2 by means of various integrated modelling codes. Iterations between systems codes and scenario modelling should eventually converge to the definition of optimum working points that are consistent with the physics guidelines. The main results of this work, which is still ongoing, are reported here. In Sec. 2, the codes used are listed and the simulation strategy adopted is briefly described. The working points produced by PROCESS are presented in Sec. 3. DEMO1 and DEMO2 assessment studies via scenario simulations are discussed in Secs. 4 and 5, respectively. Conclusions on this work at its present stage are given in Sec. 6.

\section{Codes and simulation strategy}

The computational tools used for these studies are:

- The 0.5-D integrated modelling code METIS [5]. METIS is an integrated modelling code that has the same inputs, outputs (time evolution, spatial profiles, equilibria) and non-linearities as other more complex modelling suites, however it uses a number of simplifying assumptions that make simulations of a full discharge possible in a very 
short computation time ( a few minutes). It is then particularly suited for systematic scenario exploration.

- The coupled core-edge code COREDIV [6]. The COREDIV code self-consistently solves 1D radial transport equations of plasma quantities and impurities in the core region and 2D multi-fluid transport in the Scrape-Off Layer (SOL).

- The 1.5-D integrated modelling codes ASTRA [7], JINTRAC [8] and CRONOS [9].

These codes compute the time and space evolution of the main plasma quantities (current density, electron and ion temperatures, electron or ion density) solving fluid equations with realistic sources. The 2D magnetic equilibria are computed self-consistently, assuming a given plasma boundary.

- The NBI modelling code NEMO [10] coupled to the orbit following MonteCarlo code SPOT [11]. These codes are used both in stand-alone computations and within CRONOS simulations

Starting from the 0-D outputs of the PROCESS [3] code for both DEMO1 and DEMO2 working points, presented in Sec. 3, the following steps have been performed:

- test of the consistency of the PROCESS working points by exploratory runs of METIS

- assessment of density and temperature profiles consistent with the working points and with first-principle particle transport models (TGLF [12], GLF23 [13]), by ASTRA

- assessment of impurity and radiation profiles consistent with the reference working points and with suitable impurity transport assumptions by COREDIV

- check of the NBI power deposition and driven current profiles with NEMO/SPOT

- global scenario assessment by 1.5-D simulations with JINTRAC and CRONOS

- sensitivity analysis for variations of selected plasma and machine parameters and of transport models (by METIS, ASTRA, COREDIV)

The assessment resulting from this procedure then provides input for successive iterations with PROCESS in order to improve the working point. This method has been applied in particular to DEMO1 and, to a lesser extent, to DEMO2. The present state of this investigation is described in Secs. 4 and 5, for DEMO1 and DEMO2, respectively.

\section{DEMO working points}

Systems codes describe the complex interactions of the large number of sub-systems as well as of the plasma that compose a fusion reactor. Owing to the number and complexity of the various interacting elements, substantial simplifications are made. This results, e.g., in a 0-D description of the plasma behaviour. The output of systems codes includes plasma 
(density, temperature, current, impurities, etc.) as well as machine (size, geometry, magnetic field, coils, blanket etc.) global parameters, and the ensemble constitutes a working point. PROCESS [3] can be used in the optimisation mode, to determine a working point that maximises a given set of parameters (that could related to physics, engineering and even economics), with a number of boundaries or constraints. For the present work, it was used to generate two working points i.e., DEMO1 and DEMO2. The net electrical power targets for these designs was 500MW in both cases, but while DEMO1 is assumed to be a near-future design based on ITER technology and is intended to be a pulsed device, DEMO2 assumes some advances in technology (e.g. slightly increased divertor performance, improved currentdrive efficiency, improved thermal plant efficiency) and physics (higher energy confinement improvement factor $\mathrm{H}$ and normalised beta limits) which allow it to be a steady-state machine. The machine parameters characterising these working points are summarised in Table 1, whereas the radial builds are shown in Fig. 1. DEMO1 is not fully ignited and retains some additional heating for burn control. The flux-swing requirements for a long pulse mean that DEMO1 is larger and has a higher aspect ratio than DEMO2, although the higher aspect ratio allows a higher field in the plasma for the assumed magnet technology. Discussion of the aspect ratio optimisation and additional details can be found in Ref. [14]. PROCESS uses simplified models to simultaneously solve for an operating point taking into account physics and technological limitations; however, this operating point must be verified through more detailed and self-consistent modelling, which is discussed in the following Sections.

\section{Pulsed DEMO scenario assessment (DEMO1)}

The main requirements characterising the chosen approach for the DEMO1 working points are: i) electrical power $\mathrm{P}_{\mathrm{el}}=500 \mathrm{MW}$, and ii) pulse length $\geq 2$ hours. These should be obtained while simultaneously fulfilling a number of technology and physics constraints that define acceptable boundaries of the parameter space, as, e.g., the maximum magnetic field on the conductor, maximum heat flux on PFCs, current drive efficiency compatible with proven or attainable technology, etc. [14]. Within this framework, the main physics issues specifically related to scenario development that have been addressed for DEMO1 are the following:

- compatibility of density profile with known physics (density limits, peaking)

- compatibility of temperature profile with 1 st principle models (turbulent transport and critical gradients). 
- plasma dilution and contamination by impurities

- compatibility of radiation with divertor protection and fusion performance

- compatibility of core and pedestal with beta limits and MHD instabilities

This analysis is by no means exhaustive, i.e., many more issues are important but have not been investigated so far, e.g.: ELMs, disruptions, runaways, fuelling, alpha confinement, transients and controls, etc. [4]

\subsection{Scenario analysis by METIS}

The METIS simulations are first presented. It has been checked that when assumptions similar to those of PROCESS are made, the results of METIS in the stationary phase are in good agreement with those of PROCESS. This is shown in Table 2. Some differences are related to the fact that METIS has to attain the H-mode computing the full discharge evolution, therefore some additional power (e.g. ECRH) is needed in the ramp-up phase, which has an impact on the discharge duration. Moreover, different assumptions on the core radiation fraction (defined as the ratio of the sum of the line radiation emitted inside the last closed magnetic surface, Bremsstrahlung and synchrotron radiation to the total heating power) can lead to substantially different fusion performance, as shown by the right column of Table 2.

For the low radiation case, magnetic equilibrium, time evolution of global quantities and radial profiles are shown in Figs. 2-4. Note that a sawtooth model, limiting the q decrease at $\mathrm{q} \sim 1$ is applied to this simulation. A sensitivity analysis has been performed with respect to 1) core radiation, 2) $\mathrm{H}$ factor, and 3 ) temperature pedestal $\mathrm{T}_{\text {ped }}$, the results being shown in Fig. 5. The decrease of the fusion power with the core radiation fraction is quantified and it is found that the flat-top duration decrease is significantly stronger. Note that when increasing $\mathrm{T}_{\text {ped }}$ at fixed $\mathrm{H}$ factor, the central temperature and thus the fusion power decreases (from $\mathrm{P}_{\text {fus }}=$ $2.14 \mathrm{GW}$ to $2.08 \mathrm{GW}$ for the low radiation case and from $1.66 \mathrm{GW}$ to $1.37 \mathrm{GW}$ for high radiation). Note also that the density profiles assumed in METIS have already been assessed by studies made with ASTRA and the $1^{\text {st }}$ principle TGLF model, yielding a typical density peaking factor $\mathrm{n}_{\mathrm{e} 0} /\left\langle\mathrm{n}_{\mathrm{e}}\right\rangle \sim 1.3$, when the pedestal density is assumed to be lower than the Greenwald limit, in agreement with experiments on ASDEX Upgrade [2]. This follows from the simulations presented in Sec. 4.3. 


\subsection{Impurity and radiation analysis by COREDIV}

The core radiation fraction, that in METIS simulations has been used as a scan parameter, has been investigated with the help of the COREDIV code [6], which selfconsistently solves $1 \mathrm{D}$ radial transport equations of plasma and impurities in the core region and 2D multi-fluid transport in the SOL. The model is self-consistent with respect to both the effects of impurities on the $\alpha$-power level and the interaction between seeded and intrinsic impurities. This interaction leads to a significant change in the intrinsic impurity fluxes and energy power balance, and it is found to be essential for a correct evaluation of the average power to the target plates. The code has been successfully benchmarked with a number of JET discharges, including the nitrogen seeded type I and type III ELMy H-mode discharges $[15,16]$ and recently, JET ILW configuration [17]. COREDIV has also been applied to ASDEX Upgrade discharges in the full W environment [18].

For DEMO, tungsten is assumed as the PFC armour material and argon (Ar) or neon $(\mathrm{Ne})$ is puffed as the seeding impurity in the divertor region. The sputtering processes of tungsten at target plates by all ions are taken into account. In addition, helium is produced in the core by the fusion reactions. Sensitivity with respect to inward velocity pinch is studied, varying the pinch velocity by means of a pinch factor $c_{p}$ (i.e., a factor multiplying the inward convective term in the particle flux). Examples of COREDIV results are shown in Fig. 6: profiles of line radiation, impurity concentrations and radiation $v s \mathrm{c}_{\mathrm{p}}$. The global parameters obtained using COREDIV are close to those proposed by PROCESS, although quantities such as the fusion power, radiated fraction and He concentration depend strongly on seeded impurities concentration and impurity velocity pinch.

It is found that in this DEMO reactor option, tungsten is responsible for most of the core radiation. The increase of seeded impurity influx (Ar, Ne) is accompanied by increase of $Z_{\text {eff }}$ and reduction of $P_{\alpha}$ and a corresponding decrease of the Q-factor (ratio of the fusion power to the externally applied heating power). This is caused by the change of the density of fuel ions due to the dilution effect of seeded impurity and helium. The total plasma radiation is high (typical radiation fractions of the order of $70 \%$ are systematically found) and depends very weakly on the argon or neon influx, with the majority of the radiation losses coming from the plasma core (>90\%), with significant tungsten contribution, above 50\% (Fig.6). This high radiation makes the H-mode operation of DEMO questionable, due to the power crossing the separatrix hardly above the $\mathrm{L}-\mathrm{H}$ mode power threshold $\left(\mathrm{P}_{\mathrm{L}-\mathrm{H}}\right)$. The situation is somehow better for argon seeding, whereas for neon it is not possible to keep the power flux through the separatrix above the threshold and simultaneously to reduce the power delivered 
to the target plates to technologically acceptable levels. It should be noted also, that the radiation in the SOL is usually small $(<10 \%)$ but sufficient to achieve high recycling or semi-detached conditions in the divertor.

In addition, the COREDIV results show that there is always a limit on admissible concentration of impurities (impurity puffing level) above which the solution of the power balance equation does not exist. This limit strongly depends on the type of the impurity (i.e., on its atomic number) and is related to the nonlinear interaction between fusion power, impurity radiation and dilution caused by impurity production due to sputtering processes. On top of this, also edge density limit (Borrass limit [19]) contributes to conditions limiting the seeded gas influx. In order to achieve acceptable conditions in the reactor with respect to the heat load level and confinement properties (H-mode), conditions with relatively strong radial transport in the SOL and reduced impurity pinch in the core region are desirable. It should be noted however, that it is very difficult to control such plasma properties externally.

\subsection{Impurity and radiation analysis by JINTRAC}

Fully predictive simulations of the DEMO scenarios evaluated in the PROCESS runs have been performed with the JINTRAC integrated suite of transport codes. The quantities simulated were plasma current, main ion (deuterium and tritium) densities, ion and electron temperatures and impurity (helium, argon and tungsten) concentration. The JETTO [20] transport code was deployed to simulate the main ions evolution, whereas the SANCO [21] transport code was deployed to deal with the impurities. For argon and tungsten, an initial concentration was assumed and the impurity inventory was kept constant throughout the simulation, whereas for helium the simulation started with a negligible particle inventory and the source was calculated from the D-T reaction rate.

A recycling factor $\mathrm{R}=1$ was assumed for main ions, argon and tungsten, whereas $\mathrm{R} \sim 0$ was assumed for helium. This is equivalent to the assumptions that the plasma can be adequately fuelled to maintain the density at a constant value, impurity accumulation is prevented and helium is pumped with high efficiency.

The same NBI particle and heat sources used in METIS where assumed for the JINTRAC simulations. For the transport coefficients (particle diffusivity D and heat conductivity $\chi$ ) analytical expressions were used and the absolute value of the transport coefficients was tuned in order to give the desired $\mathrm{H}$ factor. The same anomalous particle diffusivity was used for both main ions and impurities and added to the neoclassical 
contribution calculated by NCLASS [22]. An anomalous particle inward pinch of the form $0.5 \mathrm{D} \mathrm{r} / \mathrm{a}^{2}$ was also introduced to obtain peaked density profiles similar to those predicted by physics based transport models as, for example, TGLF [12]. The transport coefficients in the edge transport barrier (ETB) were tuned to provide a density at the top of the ETB of $\sim 810^{19}$ $\mathrm{m}^{-3}$ and a temperature of $\sim 5-7 \mathrm{keV}$. The core transport was then tuned to obtain a stationary simulation. It should be noted that the stability of fully predictive simulations performed is extremely sensitive to the level of heat transport assumed. Small changes in the heat conductivity, resulting in an energy confinement time only slightly lower than required, caused a radiative collapse of the discharge and a total loss of the plasma performance.

Finally, radiation profiles have been computed by the SANCO impurity code [21] within the full JINTRAC discharge simulations, with results in basic agreement with those of COREDIV when similar assumptions are made. Examples of density profiles of the three main impurities are shown in Fig. 7(a), whereas radiated power density profiles are shown in Fig. 7(b).

\subsection{Transport analysis by ASTRA/TGLF}

In all the simulations presented so far, heat transport was included by fixing the $\mathrm{H}$ factor and assuming analytical shapes for the heat diffusivity. In order to check the consistency of the predicted performance with physics based transport models, simulations have been carried out with ASTRA [7] including the TGLF [12] turbulence/transport model (a quasi-linear gyro-Landau-fluid model tuned on the results of non-linear gyrokinetic simulations). In addition, the temperature pedestal has been varied and a scan of the Argon concentration has been carried out, which corresponds to a scan of the core radiation and plasma dilution, keeping $\mathrm{W}$ concentration fixed at $310^{-5}$.

The main results are shown in Fig. 8. An $\mathrm{H}$ factor in agreement with that of the PROCESS working point $(\mathrm{H}=1.1)$ is obtained for $\mathrm{T}_{\text {ped }}=9 \mathrm{keV}$, a value significantly higher than in Fig. 4. An analogous result is obtained by JINTRAC. Whether this high pedestal value is compatible with pedestal MHD limits and with energy release to the divertor by the ELMs is an important subject of investigation. The temperature and density peaking factors are found to be limited by critical gradients (middle panel of Fig. 8), which for the temperatures are found in the range $4<\mathrm{R} / \mathrm{L}_{\mathrm{T}}<6$, from core to edge. Higher normalized gradient values could be attained if additional stabilizing mechanisms act on turbulence. In the case shown here, the main one is plasma dilution due to impurities, plus the effect of a large Shafranov 
shift due to the alpha particles pressure. However, it has been recently found that improved stabilization has to be expected by the presence itself of the alpha particles [23]. This could lead to higher core normalized gradients and thus better performance (in particular as the requirement of a high pedestal top pressure could be relaxed).

For the electron density profile, the normalized gradient is found in the range $\mathrm{R} / \mathrm{L}_{\mathrm{n}} \sim 1$ 2 , consistent with a theoretically predicted ratio $\left(\mathrm{R} / \mathrm{L}_{\mathrm{n}}\right) /\left(\mathrm{R} / \mathrm{L}_{\mathrm{T}}\right) \sim 0.2-0.3$. The reason why such a value for $\mathrm{R} / \mathrm{L}_{\mathrm{n}}$ is attained is the low collisionality range in which DEMO operates [24]. Upon increasing the collisionality by a factor of $\sim 10$, the peaking factor is reduced to almost 1 (i.e. flat density profile), as discussed in [2], a result that has been obtained in a set of simulations with fixed temperature profiles. Electro-magnetic effects are also responsible for a slight reduction of peaking, as expected. In Fig. 9, the density peaking factor is shown as a function of the temperature peaking factor, for a set of simulations with different impurity contents. The straight line is the fit $\mathrm{p}_{\mathrm{n}}=1+0.24\left(\mathrm{p}_{\mathrm{T}}-1\right)$ between density peaking $\mathrm{p}_{\mathrm{n}}$ and temperature peaking $\mathrm{p}_{\mathrm{T}}$. It is clear that this proportionality determines one element that could help in increasing the fusion power, i.e. the synergistic effect of increasing $T_{e} / n_{e}$ peaking factor.

The Argon scan gives a quantitative estimate of the significant impact of core radiation and plasma dilution (which is in fact the dominant effect here) on the fusion performance of the working point (Fig. 8, right plot). Dilution reduces the fusion power, albeit at the same time it reduces transport via the effect on the ITG. The net effect is anyway to reduce fusion power in a significant way, questioning the actual choice of the seeded impurities and requiring optimization. An increased pedestal leads to more margin of operation of course, but again the pedestal stability is always an issue when increasing this parameter.

\section{Steady-state DEMO scenario assessment (DEMO2)}

For the steady-state DEMO concept (DEMO2), the same electrical power as for DEMO1 is assumed (500 MW), however the challenging requirement of full non-inductive current sustainment is also imposed. Although not used here as an optimization parameter, the fusion gain $\mathrm{Q}$ tends to decrease in this type of scenario, because of the large CD power required to sustain the discharge, therefore care must be taken to optimize the bootstrap fraction in order to minimize the $\mathrm{CD}$ power and keep $\mathrm{Q}$ at a reasonable level (e.g., > 10). The main scenario issues specific to DEMO2 that should be considered are the following: 
- bootstrap current optimisation

- non-inductive CD optimisation

- possible use of ITB regimes

- access to fully non-inductive scenario (combining the previous points)

- $\quad$ high beta MHD limits (e.g., acceptable $\beta_{N} / 4$ li range)

As discussed in Sec. 3, the DEMO2 working point obtained by PROCESS and considered at this stage is characterised by a heating power of $135 \mathrm{MW}$ and a marginally acceptable fusion gain, $\mathrm{Q}=16$.

\subsection{Scenario analysis by METIS}

As for DEMO1, the working point has been checked by METIS simulations, again with a core radiation scan (see Table 3 and Figs. 10-12). The fusion performance has been reproduced, however not in fully non-inductive conditions. This is mainly related to differences in the bootstrap fraction, which are due to differences in the models used, a subject that is now being actively investigated. The plasma performance is found to decrease with increasing core radiation. This can be partially compensated by increasing the CD power, which also helps to attain the fully non-inductive regime. The drawback in this case is a substantial decrease of Q (see right column of Table 3). Note that a degree of freedom in this type of scenarios is given by the radial location of the NBI driven current. In these METIS simulations, an off-axis CD has been used, in order to produce a q profile without a $\mathrm{q}$ $=1$ surface and compatible with high confinement (see Fig. 11). An ad-hoc CD efficiency $\gamma_{\mathrm{CD}}$ 0.4-0.45 A. $\mathrm{W}^{-1} \cdot 10^{20} \mathrm{~m}^{-2}$ has been assumed, which could be compatible, e.g., with a $1.5 \mathrm{MeV}$ NBI system. The CD efficiency, profile, as well as the bootstrap fraction, are critical for this type of scenario. NBI CD is discussed in more detail in Sec. 5.2.

Concerning the MHD stability, it should be stressed that this scenario is characterised by a high confinement improvement $(\mathrm{H}=1.3)$ and a mildly reversed q profile, therefore high beta and low internal inductance. As shown by Fig. 12 , in this case $\beta_{\mathrm{N}}$ is larger than $41_{i}$, which implies that ideal no-wall MHD limits could be attained and active controls should be possibly employed. 
In order to access to fully non inductive scenarios, DEMO2 relies both on bootstrap current and current driven by auxiliary systems. The scenario analysed in this section by means of METIS 0.5-D integrated modelling code is presented in Table 3 as the "low radiation" case. All the auxiliary heating and current drive power (135 MW) is provided by NBI, which in the METIS code is represented by 2 injection lines, each carrying $67.5 \mathrm{MW}$. The total auxiliary power in DEMO2 is at least twice that of DEMO1, due to the necessity of driving more non inductive current. Injection geometry similar to that of ITER has been assumed as for the DEMO1 case.

In this section, profiles of driven current density by NBI for different beam parameters are shown in order to highlight the impact of different NBI set-up choices. Results are shown for stationary plasma conditions during the flat-top phase.

Figure 13(a) shows the current density profiles driven by beams with different energies, from $750 \mathrm{keV}$ to $2 \mathrm{MeV}$. The reference scenario relies on $1 \mathrm{MeV}$ beams, although options at lower or higher beam energy, with an impact on both CD efficiency and overall wall-plug efficiency [25], are still being considered in the scientific community. An investigation of a DEMO scenario with $2 \mathrm{MeV}$ NBI is carried out in [26], including a comparison with $1 \mathrm{MeV}$ NBI case. A study on the requirements and consequent design of a 1.5 MeV NBI for DEMO is presented in [27].

The maximum of the current density profile only slightly moves towards the centre when increasing the energy, while, with respect to the reference NBI energy $(1 \mathrm{MeV})$, there is an increase of total driven current of $\sim 30 \%$ moving to $2 \mathrm{MeV}$, or a decrease of $\sim 15 \%$ moving to $750 \mathrm{keV}$. While a possible reduction of the beam energy is not a major issue for the DEMO1 pulsed scenario, it could strongly affect the achievement of DEMO2 steady state scenario, unless the total power coupled to the plasma is increased. Note however that this could be obtained, as stated above, by a better tuning of the wall-plug efficiency (easier at lower beam energies), keeping constant the input electrical power to the injector system. Assuming instead some future technological advancement one could think of increasing the NBI energy in order to increase the current drive efficiency (more accurate values are then estimated using NEMO/SPOT inside CRONOS).

The vertical tilt of the beam line is a parameter which can be modified in the METIS code, and a tilt of the beam injection line with respect to the horizontal plane can be simulated. Figure 13(b) shows the effect of different vertical tilts on the current density profile driven by the beams. The values reported in the legend of Fig. 13(b) are the normalized coordinates $[0=$ centre, $1=\mathrm{LCMS}]$ where the beam intersects the vertical axis 
which passes through the plasma magnetic axis. The value reported is applied to both injectors in METIS code. The modification of the vertical tilt is a method to induce current centred at different radial positions. In particular, with 0 vertical tilt the driven current density is peaked at the centre, while for a tilt of 0.5 the maximum of the curve moves to almost 0.6 in normalized poloidal flux coordinate. The reference scenario presented in Table 3 has the two beam lines with vertical tilt of 0.20 and 0.41 respectively (shown in Fig. 13(b) in dotted red line). This combination of different vertical tilts for the two beams makes the profile broader and, being the driven current off-axis, helps in maintaining the mildly reversed $\mathrm{q}$ profile. The total driven current does not change significantly with the tilt of the beam lines, therefore the effect of the vertical tilt is negligible on the current drive efficiency.

\subsection{NBI current drive analysis by CRONOS}

The DEMO2 reference scenario has also been studied with the CRONOS code, including the NEMO/SPOT NBI module [10]. In order to assess the CD efficiency that can be expected in this type of scenario, NBI injection geometry similar to that of ITER has been assumed. The alpha particle distribution has been computed by means of the orbit following MonteCarlo code SPOT [11]. The density profile computed by METIS for the low-radiation case has been assumed. Time evolution has been computed for the temperatures and for the current density, for $50 \mathrm{~s}$ during the flat-top phase. Different NBI injection geometries have been tested, yielding more or less off-axis driven current profiles. Two examples are shown in Fig. 14, together with the METIS case. Note that in METIS the NBI CD efficiency was adjusted in order to match that of PROCESS, whereas in the CRONOS simulations it has been computed from first principle theory. Typical efficiencies of the order of 0.3-0.4 A.W${ }^{1} .10^{20} \mathrm{~m}^{-2}$ have been obtained for beam injection energy of $1 \mathrm{MeV}$.

The heat diffusivity has been computed in two different ways: i) as in METIS, by the Kiauto model, i.e., an analytical shape and a renormalisation that yields the plasma energy corresponding to the H-mode scaling [9]; ii) the GLF23 first principle transport model [13]. A comparison of the various current sources for the two different heat transport models considered is shown in Fig. 15. Both NBI and (especially) bootstrap currents are found to be sensitive to the heat transport model used. Finally, the current associated to the alpha particle distribution has also been computed by the SPOT code. The profiles of the alpha current density are shown in Fig. 16, both with and without the electron shielding effect (which 
corresponds to the effective current density source and the ion current density, respectively). The total alpha-driven current is found to be $0.34 \mathrm{MA}$, i.e., $1.8 \%$ of the total current. As for ITER, it plays a minor role in the establishment of the overall current density profile.

\subsection{Impurity and radiation analysis by JINTRAC}

An evaluation of the DEMO2 working point has also been carried out using the JINTRAC suite of codes. The same process and inputs as described in Sec. 4.3 have been used, with JETTO simulating the main ions, SANCO simulating the impurity ions and transport coefficients being tuned to give the desired $\mathrm{H}$ factor. This led to a temperature at the $\mathrm{ETB}$ of $\sim 9 \mathrm{keV}$.

As with DEMO1, the DEMO2 simulations were sensitive to the level of heat transport used: with lower confinement either the plasma would suffer a radiative collapse or a higher level of input power than in the PROCESS run was required.

Impurity radiation profiles have been produced by the SANCO code for a stable DEMO2 simulation. The total amount of radiation is higher than in the DEMO1 case, reflecting the higher value of $\mathrm{Z}_{\text {eff }}$ in the DEMO2 working point. The simulations were also very sensitive to the level of Tungsten included in the initial conditions. The density profiles for the impurities are shown in Fig. 17(a) and the radiation profiles are shown in Fig 17(b).

\section{Summary and conclusions}

As a result of this scenario assessment, a number of issues of the DEMO1 and DEMO2 scenarios have been identified and quantified. Depending on their nature and impact on the scenario, they require further studies with more sophisticated codes, improvement of the experimental knowledge by dedicated experiments, or modification of the working points, a work that is ongoing. The main issues of the two scenarios can be summarised as follows.

Fusion performance: the fusion power sensitively depends on assumptions on impurity transport and temperature pedestal. Access to pedestal temperatures of the order of $10 \mathrm{keV}$ seems necessary for these working points if stiff transport models (such as GLF23 and TGLF) are assumed.

Density profile: using the assumption $\mathrm{n}_{\mathrm{ped}}<\mathrm{n}_{\text {Greenwald, }}$ in agreement with experiments on ASDEX Upgrade [2], the 1st principle transport model TGLF yields a density peaking factor 
$\sim 1.3$ (owing to low collisionality, the electro-magnetic effects being weak). This yields Greenwald fractions $>1$, which is very favourable for plasma performance. However, the confinement properties of such discharges should be extensively studied and assessed.

Temperature profile: transport simulations with the TGLF model have shown that temperature peaking factors $\sim 2$ are compatible with critical gradients limitations associated to turbulence. Larger peaking factors would be favourable but do not appear realistic. However, fast-particle and beta stabilizing effects have not yet been included in the assessment.

Radiation, plasma dilution, impurities: self-consistent impurities/radiation evaluation is one of the most critical issues. Scanning the core radiation fraction from low $(\sim 0.3)$ to high $(\sim 0.75)$ values appears a safe option in order to determine a working point with sufficient margins. The fusion power is found to decrease with core radiation by $\sim 30 \%$ in this range, mostly due to dilution from radiating impurity. Confinement scaling laws in highly radiating regimes should be assessed, as well as impurity transport models.

Discharge duration (DEMO1): a flat-top time of the order of 2 hours can be obtained, and optimised by a careful use of H\&CD in the ramp-up phase. However, evaluation by more sophisticated codes is necessary (free-boundary equilibrium codes), since the flat-top time sensitively depends on the plasma performance (therefore on radiation, pedestal, etc.)

MHD (DEMO1): large sawteeth are expected, which implies that NTM must be controlled, as in the baseline scenario of ITER.

$M H D(D E M O 2): \beta_{\mathrm{N}}>41_{\mathrm{i}}$ systematically in these scenarios. This issue should be addressed by MHD analysis and appropriate design of the control systems.

Bootstrap current (DEMO2): bootstrap fraction $\mathrm{f}_{\mathrm{bs}}>50 \%$ is needed, but this seems intrinsically difficult to attain for $\mathrm{q}_{95}<4$, which is in turn required for energy confinement Non-inductive CD optimisation (DEMO2): NBI CD efficiency should be carefully assessed for the DEMO2 parameter range. At the present stage, for a NBI injection energy of $1 \mathrm{MeV}$, the current drive efficiency is found to be of the order of $\gamma_{\mathrm{CD}} \sim 0.35 \mathrm{~A} . \mathrm{W}^{-1} 10^{20} \mathrm{~m}^{-2}$, i.e., of the same order as that expected for ITER. The NBI system is a powerful tool for profile and scenario control, but the NBI geometry has to be compatible with the machine design.

In conclusion, the work presented here is just a step of a long-term $R \& D$ work ongoing on the development of DEMO scenarios in the EU. It shows the basic problems encountered and the research lines along which solutions should be found. Of course, the physics assessment of DEMO scenario is expected to be substantially improved, in many respects, by the experiments that will be carried on in ITER. 


\section{Acknowledgements}

This work was supported by EURATOM and carried out within the framework of the European Fusion Development Agreement and of the EUROfusion Consortium and has received funding from the European Union's Horizon 2020 research and innovation programme under grant agreement number 633053. The views and opinions expressed herein do not necessarily reflect those of the European Commission. 


\section{References}

[1] Federici G. et al 2014 Fusion Engin. and Design 89882

[2] Zohm H. et al. 2013 Nucl. Fusion 53073019

[3] Ward D. Plasma Phys. Contr. Fusion 201052 124033; Kovari M. et al 2014 Fusion Engin. and Design 893054

[4] Wenninger R. et al 2014 25th IAEA Fusion Energy Conference, PPC/P4-19 (St. Petersburg, Oct. 13-18, 2014).

[5] Artaud J.F. et al 2005 32nd EPS Conf. on Control. Fusion and Plasma Phys., ECA Vol. 29C, P1.035

[6] Zagórski R. et al 2013 Nucl. Fusion 53073030

[7] Pereverzev G.V. and Yushmanov Y. P., 1991 Technical Report IPP 5/42, IPP, Garching, Germany; Fable E. et al 2013 Plasma Phys. Control. Fusion 55124028

[8] Romanelli M. et al 2014 Plasma and Fusion Research 934030231

[9] Artaud J.F. et al 2010 Nucl. Fusion 50043001

[10] Schneider M. et al 2011 Nucl. Fusion 51063019

[11] Schneider M. et al 2005 Plasma Phys. Contr. Fusion 472087

[12] Staebler G.M. et al 2007 Phys. Plasmas 14055909

[13] Waltz R.E. et al 1997 Phys. Plasmas 42482

[14] Kemp R. et al 2014 25th IAEA Fusion Energy Conference, FIP/3-3 (St. Petersburg, Oct. 13-18, 2014)

[15] Rapp J. et al 2005 J. Nucl. Mater. 337-339 826

[16] Zagórski R. et al 2009 J. Nucl. Mater. 390-391 404

[17] Ivanova-Stanik I. et al 2014 Contrib. Plasma Phys. 54442

[18] Zagórski R. et al 2012 Contrib. Plasma Phys. 52379

[19] Borrass K. 1991 Nucl. Fusion 311035

[20] Cenacchi G. and Taroni A. 1988 JET-IR(88)03.

[21] Lauro-Taroni L. et al 1994 Proc. 21st EPS I, 102

[22] Houlberg W.A. et al 1997 Phys. Plasmas 43230

[23] Citrin J. et al 2013 Phys. Rev. Lett. 111155001

[24] Angioni C. et al. 2003 Phys. Rev. Lett. 90 205003; Fable E. et al. 2010 Plasma Phys. Control. Fusion 52015007

[25] Franzen P. and Fantz U., 2014 Fusion Engin. and Design 892594

[26] Garcia J. et al 2008 Nucl. Fusion 48075007

[27] Surrey E. et al 2012 Fusion Engin. and Design 87373 


\begin{tabular}{|l|c|c|}
\hline & DEMO1 & DEMO2 \\
\hline major/minor radius $(\mathrm{m})$ & $9.25 / 2.64$ & $8.15 / 2.98$ \\
\hline toroidal field $(\mathrm{T})$ & 6.8 & 5.04 \\
\hline plasma current $(\mathrm{MA})$ & 18.56 & 19.85 \\
\hline heating power $(\mathrm{MW})$ & 50 & 135 \\
\hline $\mathrm{f}_{\text {Greenwald }} / \mathrm{H}$ factor & $1.2 / 1.1$ & $1.2 / 1.3$ \\
\hline$\beta_{\mathrm{N}} / \beta_{\mathrm{p}}$ & $2.47 / 1.08$ & $3.44 / 1.18$ \\
\hline$\left\langle\mathrm{n}_{\mathrm{e}}\right\rangle\left(10^{20} \mathrm{~m}^{-3}\right) /$ peaking & $0.91 / 1.3$ & $0.77 / 1.3$ \\
\hline$\left\langle\mathrm{T}_{\mathrm{e}}\right\rangle(\mathrm{keV}) /$ peaking & $14.0 / 2.0$ & $15.5 / 2.0$ \\
\hline$\left\langle\mathrm{T}_{\mathrm{i}}\right\rangle(\mathrm{keV}) /$ peaking & $14.0 / 2.0$ & $15.5 / 2.0$ \\
\hline $\mathrm{P}_{\text {fus }}(\mathrm{MW})$ & 2119 & 2104 \\
\hline$\tau_{\text {flat-top }}(\mathrm{h})$ & 1.85 & $\infty$ \\
\hline
\end{tabular}

Table 1 : main parameters of the two working points computed by means of PROCESS

\begin{tabular}{|l|c|c|c|}
\hline \multirow{2}{*}{} & PROCESS & METIS & METIS \\
\cline { 2 - 4 } & DEMO1 & $\begin{array}{c}\text { DEMO1 } \\
\text { low radiation }\end{array}$ & $\begin{array}{c}\text { DEMO1 } \\
\text { high radiation }\end{array}$ \\
\hline $\mathrm{R}(\mathrm{m}) / \mathrm{a}(\mathrm{m}) /$ aspect ratio & $9.25 / 2.64 / 3.5$ & $9.25 / 2.64 / 3.5$ & $9.25 / 2.64 / 3.5$ \\
\hline volume $\left(\mathrm{m}^{3}\right) / \mathrm{q}_{95}$ & $2009 / 3.0$ & $2009 / 3.1$ & $2009 / 3.1$ \\
\hline toroidal field $(\mathrm{T})$ & 6.8 & 6.8 & 6.8 \\
\hline plasma current $(\mathrm{MA})$ & 18.56 & 18.56 & 18.56 \\
\hline heating power $(\mathrm{MW})$ & 50 & 50 & 50 \\
\hline $\mathrm{f}_{\text {Greenwald }}$ & 1.2 & 1.2 & 1.2 \\
\hline $\mathrm{Z}_{\text {eff }}$ & 2.82 & 2.26 & 2.31 \\
\hline$\beta_{\mathrm{N}} / \beta_{\mathrm{p}}$ & $2.47 / 1.08$ & $2.60 / 1.16$ & $2.11 / 0.98$ \\
\hline$\left\langle\mathrm{n}_{\mathrm{e}}\right\rangle\left(10^{20} \mathrm{~m}^{-3}\right) /$ peaking & $0.91 / 1.3$ & $0.91 / 1.3$ & $0.91 / 1.3$ \\
\hline$\left\langle\mathrm{T}_{\mathrm{e}}\right\rangle(\mathrm{keV}) /$ peaking & $14.0 / 2.0$ & $13.8 / 2.2$ & $11.6 / 2.2$ \\
\hline$\left\langle\mathrm{T}_{\mathrm{i}}\right\rangle(\mathrm{keV}) /$ peaking & $14.0 / 2.0$ & $14.5 / 2.3$ & $12.4 / 2.2$ \\
\hline$\tau_{\mathrm{E}}(\mathrm{s}) / \mathrm{H}$ factor & $4.16 / 1.1$ & $3.97 / 1.1$ & $5.79 / 1.1$ \\
\hline $\mathrm{P}_{\text {loss }}(\mathrm{MW})$ & 303 & 318 & 184 \\
\hline $\mathrm{P}_{\text {fus }}(\mathrm{MW}) / \mathrm{Q}$ & $2119 / 42$ & $2144 / 43$ & $1658 / 33$ \\
\hline $\mathrm{P}_{\text {sync }}(\mathrm{MW})$ & 54 & 47 & 30 \\
\hline $\mathrm{P}_{\text {rad }}(\mathrm{core}) / \mathrm{P}_{\text {heat }}$ & 0.36 & 0.35 & 0.77 \\
\hline $\mathrm{f}_{\mathrm{bs}} / \mathrm{f}_{\mathrm{ni}}$ & $0.32 / 0.44$ & $0.30 / 0.44$ & $0.24 / 0.37$ \\
\hline flux swing $(\mathrm{Wb}) / \tau_{\text {flat-top }}(\mathrm{h})$ & $753 / 1.85$ & $753 / 2.71$ & $753 / 1.25$ \\
\hline & & & \\
\hline
\end{tabular}

Table 2 : main global parameters of the DEMO1 working point computed by means of PROCESS and of METIS (with two different core radiation levels) 


\begin{tabular}{|l|c|c|c|}
\hline & PROCESS & METIS & METIS \\
\cline { 2 - 4 } & DEMO2 & $\begin{array}{c}\text { DEMO2 } \\
\text { low rad. }\end{array}$ & $\begin{array}{c}\text { DEMO2 } \\
\text { high rad., high P }\end{array}$ \\
\hline major/minor radius (m) & $8.15 / 2.98$ & $8.15 / 2.98$ & $8.15 / 2.98$ \\
\hline elongation / triangularity & $1.61 / 0.33$ & $1.61 / 0.33$ & $1.61 / 0.33$ \\
\hline toroidal field $(T)$ & 5.04 & 5.04 & 5.04 \\
\hline plasma current $(\mathrm{MA})$ & 19.85 & 19.85 & 19.85 \\
\hline heating power $(\mathrm{MW})$ & 135 & 135 & 210 \\
\hline $\mathrm{f}_{\text {Greenwald }}$ & 1.2 & 1.2 & 1.2 \\
\hline $\mathrm{H}$ factor & 1.3 & 1.3 & 1.3 \\
\hline$Z_{\text {eff }}$ & 3.28 & 2.22 & 2.26 \\
\hline$\beta_{\mathrm{N}} / \beta_{\mathrm{p}}$ & $3.44 / 1.18$ & $3.56 / 1.21$ & $3.33 / 1.10$ \\
\hline$\left\langle\mathrm{n}_{\mathrm{e}}\right\rangle\left(10^{20} \mathrm{~m}^{-3}\right) /$ peaking & $0.77 / 1.3$ & $0.77 / 1.3$ & $0.77 / 1.3$ \\
\hline$\left\langle\mathrm{T}_{\mathrm{e}}\right\rangle(\mathrm{keV}) /$ peaking & $15.5 / 2.0$ & $14.9 / 2.3$ & $13.5 / 2.3$ \\
\hline$\left\langle\mathrm{T}_{\mathrm{i}}\right\rangle(\mathrm{keV}) /$ peaking & $15.5 / 2.0$ & $14.9 / 2.3$ & $14.2 / 2.4$ \\
\hline$\tau_{\mathrm{E}}(\mathrm{s})$ & 3.96 & 3.72 & 4.3 \\
\hline $\mathrm{P}_{\text {fus }}(\mathrm{MW}) / \mathrm{Q}$ & $2104 / 16$ & $2111 / 16$ & $1837 / 9$ \\
\hline $\mathrm{P}_{\text {sync }}$ & 34 & 28 & 22 \\
\hline $\mathrm{P}_{\text {rad }}(\mathrm{core}) / \mathrm{P}_{\text {heat }}$ & 0.49 & 0.33 & 0.74 \\
\hline $\mathrm{f}_{\mathrm{bs}} / \mathrm{f}_{\mathrm{ni}}$ & $0.56 / 1.00$ & $0.40 / 0.84$ & $0.38 / 1.00$ \\
\hline
\end{tabular}

Table 3 : main global parameters of the DEMO2 working point computed by means of PROCESS and of METIS (with two different core radiation and heating power levels) 

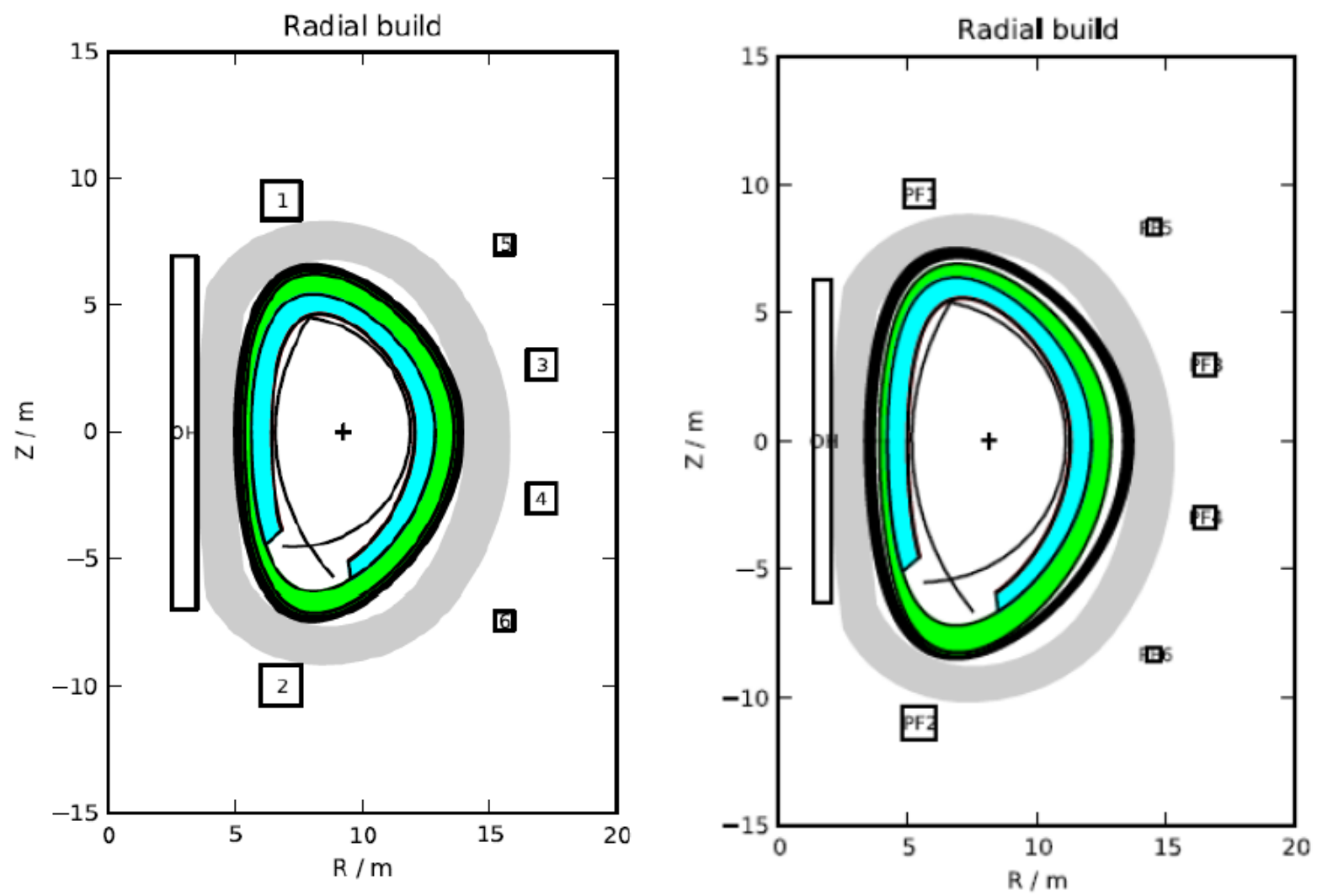

Fig. 1: Illustration of the cross-section of DEMO1 (left) and DEMO2(right), taken from PROCESS output 


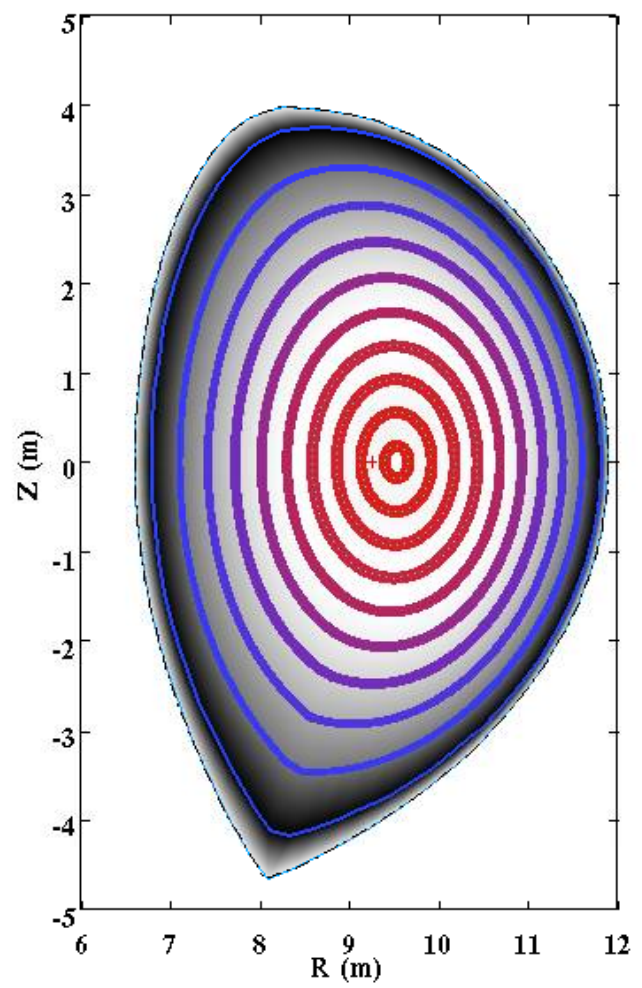

Fig. 2 : DEMO1: METIS magnetic equilibrium in the stationary phase (low radiation case)
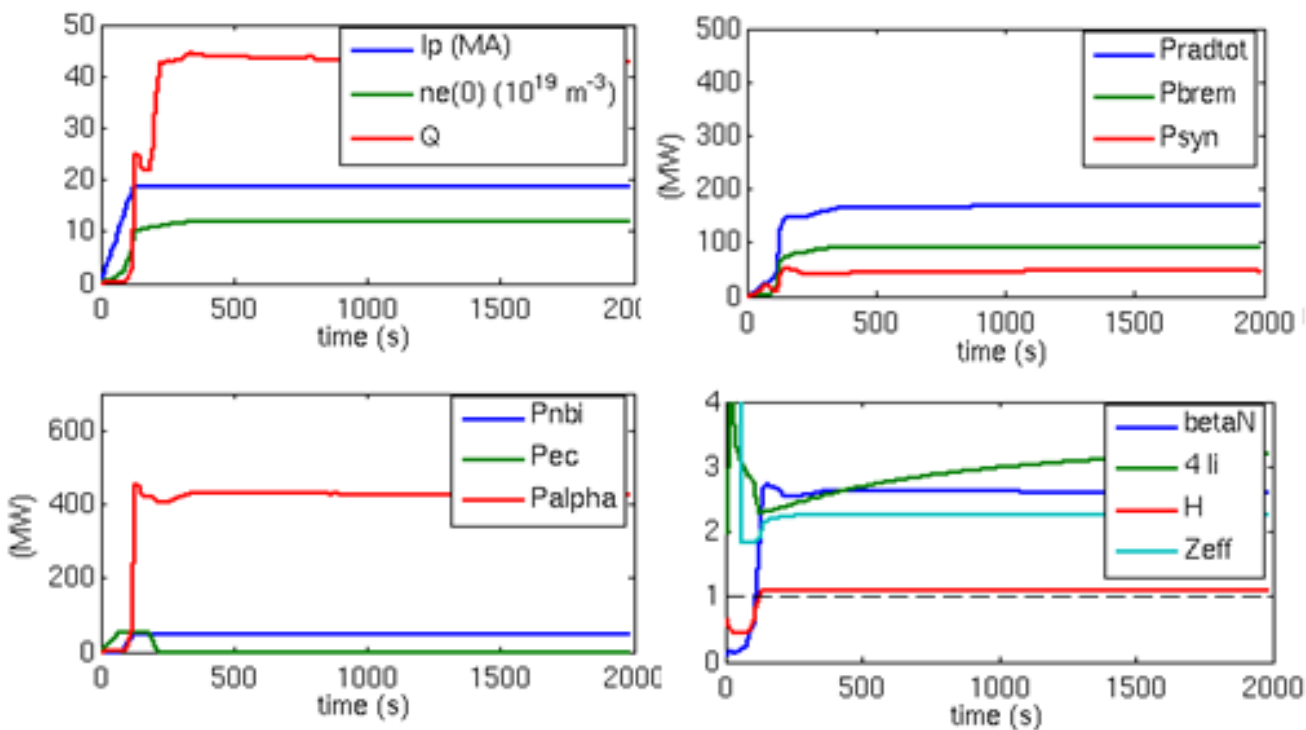

Fig. 3 : Time evolution of plasma current, central density and $Q$ factor (top left), NBI, ECRH and alpha powers (bottom left); radiated powers (top right); $\beta_{N}, 4 l_{i}, H$ factor and $Z_{\text {eff }}$ (bottom right). 

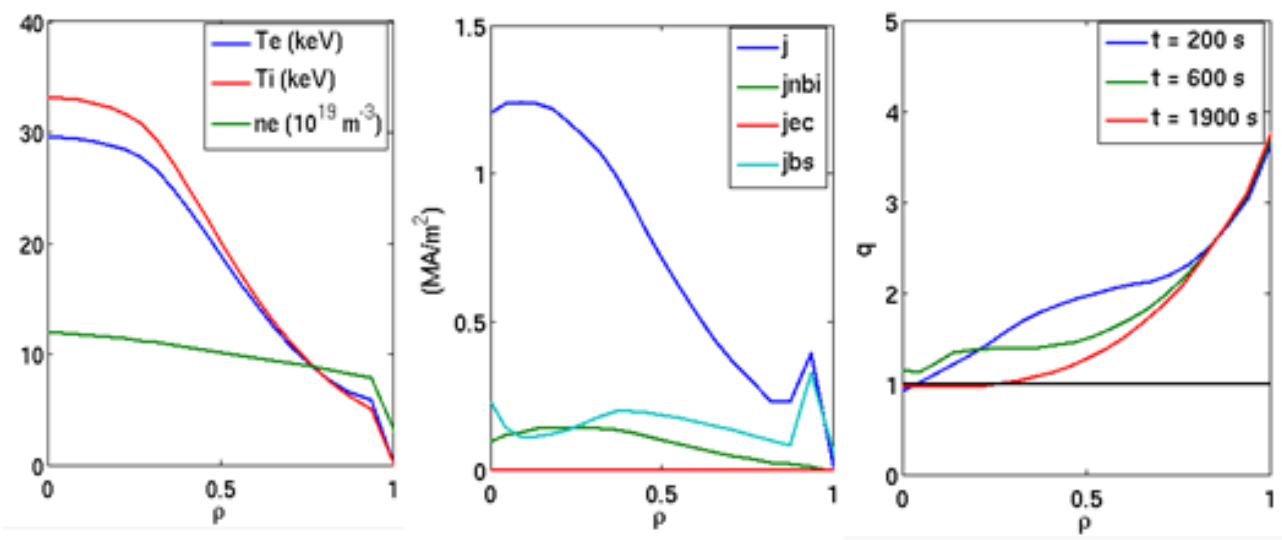

Fig. 4 : Radial profiles in the stationary phase. Electron and ion temperature, electron density (left), total current density and NBI, EC, bootstrap current density sources (middle), safety factor profile at three different times (right). A sawtooth model limiting the q decrease at $q \sim 1$ is applied to this simulation.
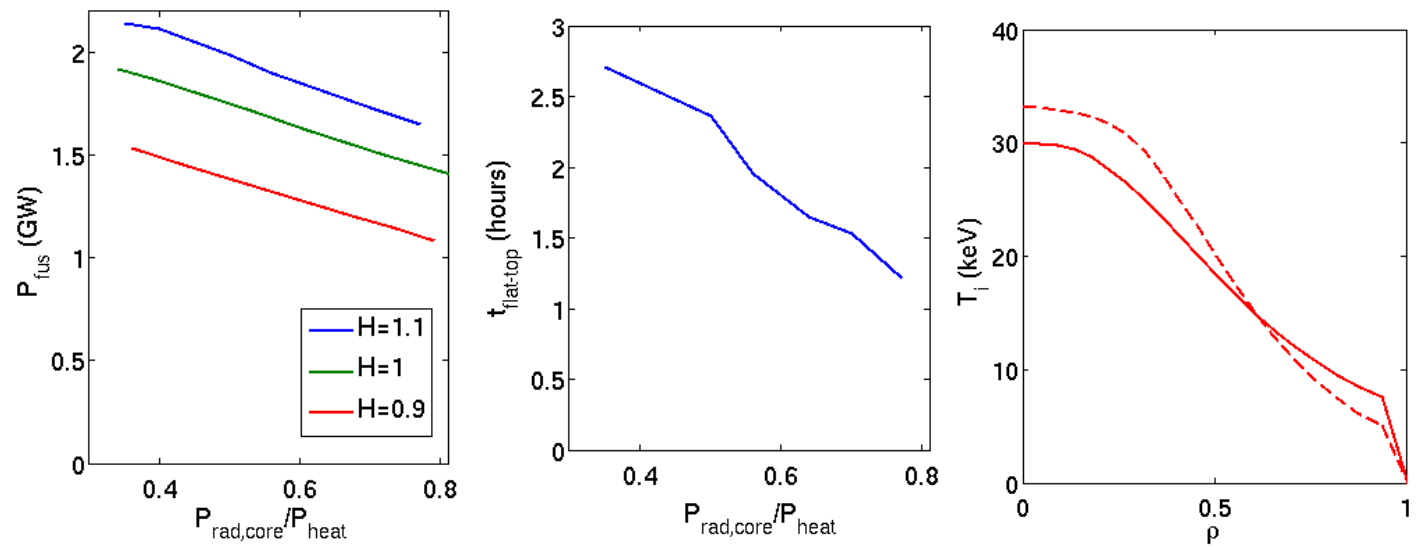

Fig. 5 : Left: fusion power versus radiation fraction. Middle: flat-top time versus radiation fraction, $(H=1.1)$. Right: ion temperature profiles for low (dashed) and high pedestal (solid) 

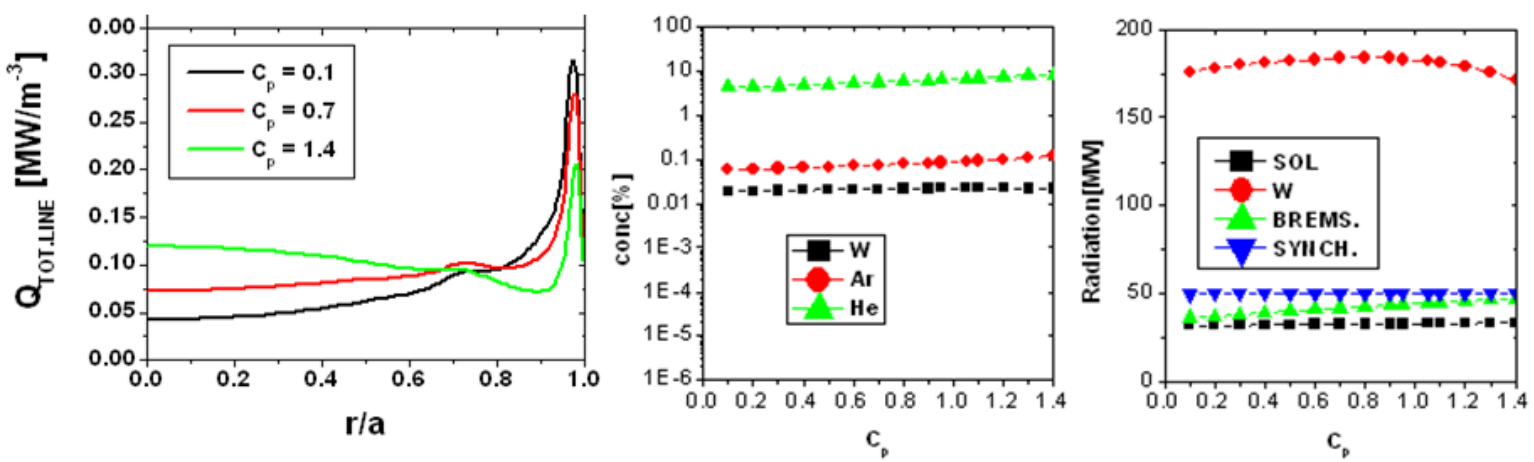

Fig. 6 : Examples of COREDIV simulation results: profiles of line radiation (left), impurity concentrations (middle) and radiated power (right) $v$ s the inward pinch factor $c_{p}$
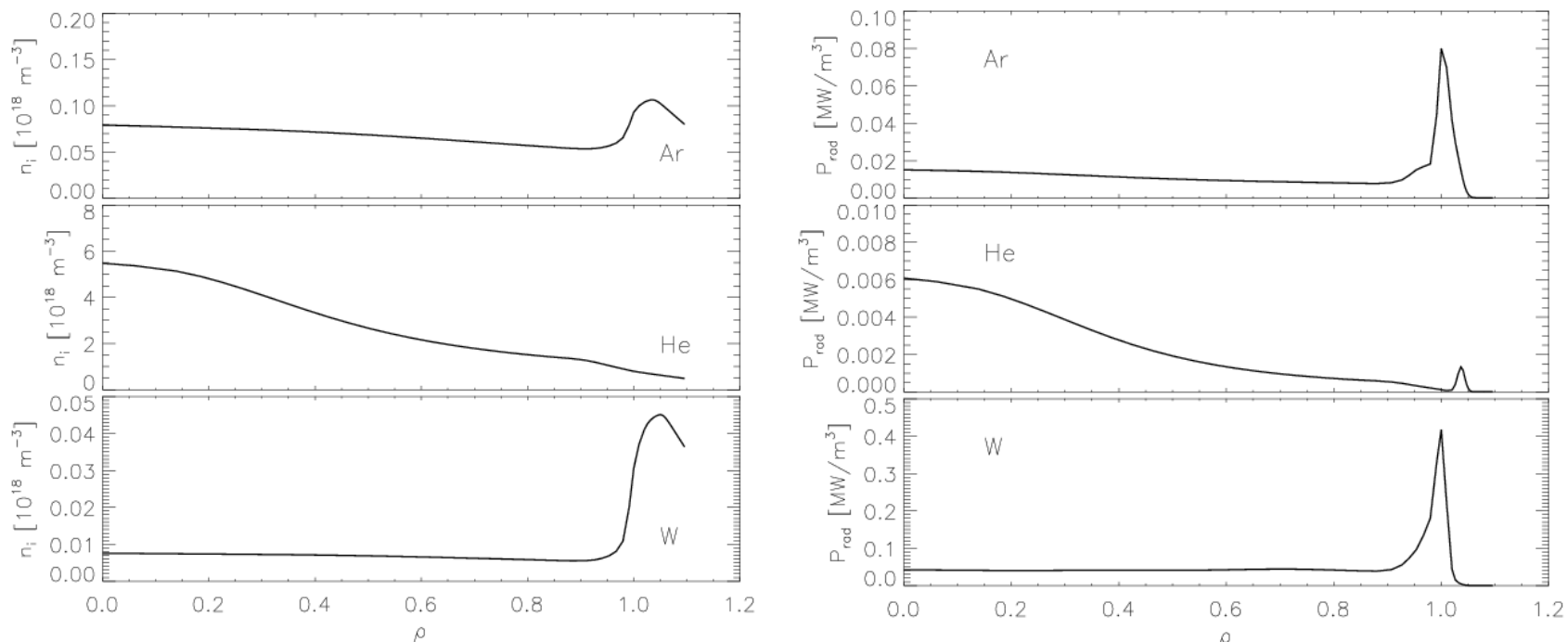

Fig. 7 : Argon, Helium and Tungsten density profiles vs normalized radius in the stationary phase of DEMO1, simulated by JINTRAC, with the SANCO impurity model (a), left; radiated power density profiles by the three impurities (b), right. 

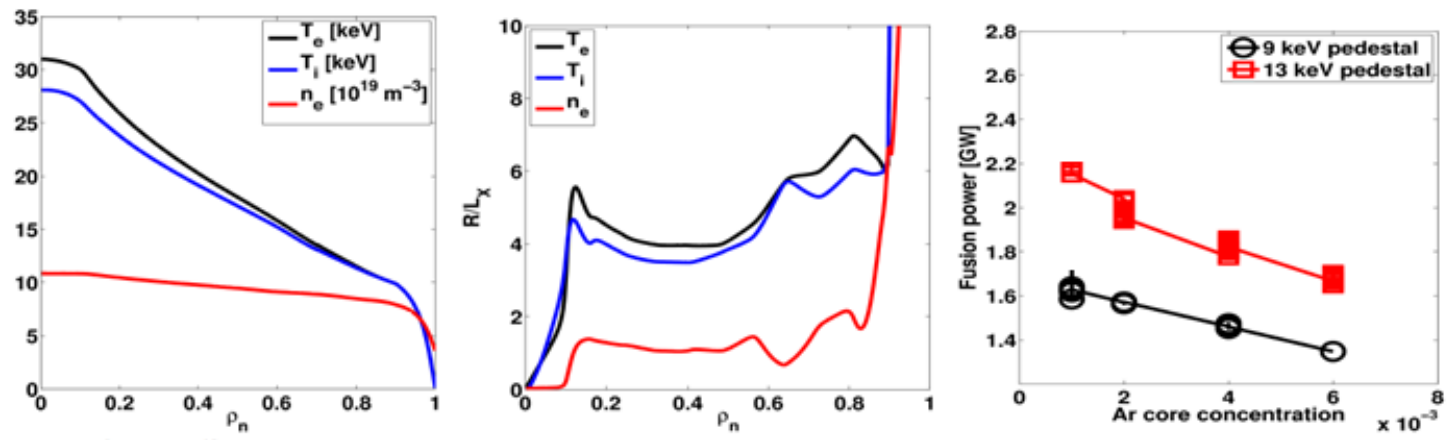

Fig. 8 : ASTRA/TGLF simualtions. Left: kinetic profiles in the stationary phase $\left(T_{\text {ped }}=9\right.$ $\mathrm{keV}$ ). Middle: gradients normalized to major radius. Right: fusion power vs Ar concentration in the core.

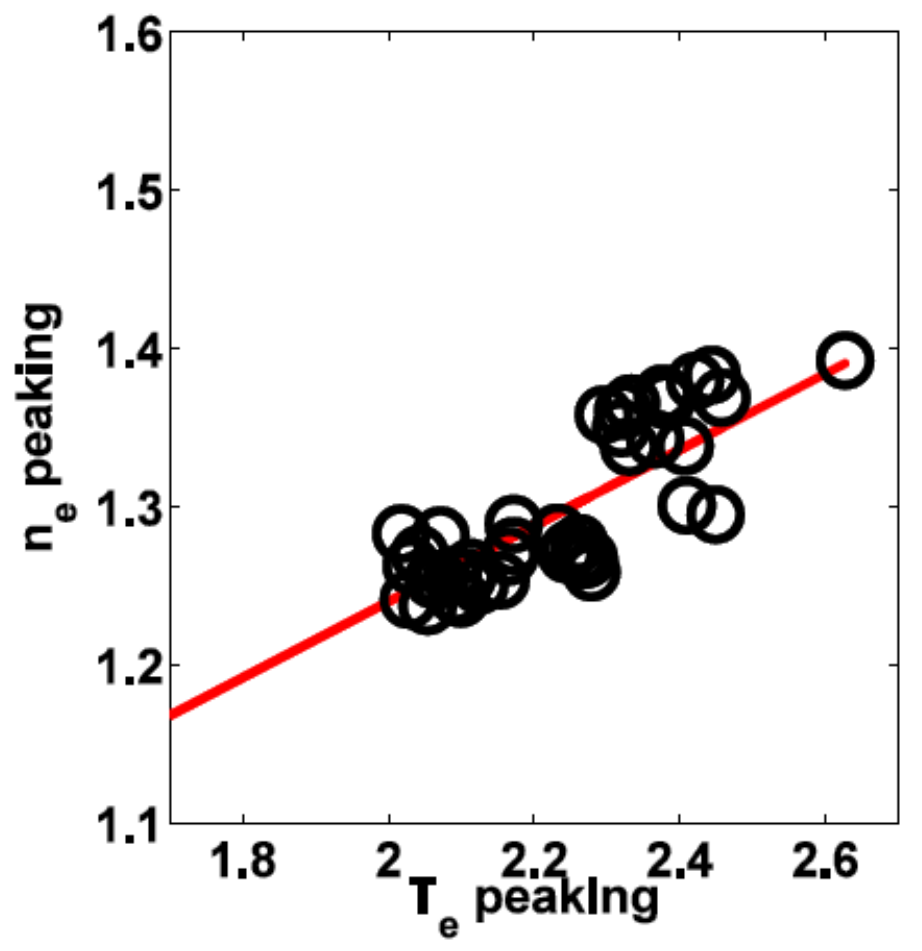

Fig. 9: DEMO1: Density peaking factor versus electron temperature peaking factor from a set of ASTRA/TGLF runs with different impurity concentrations. 


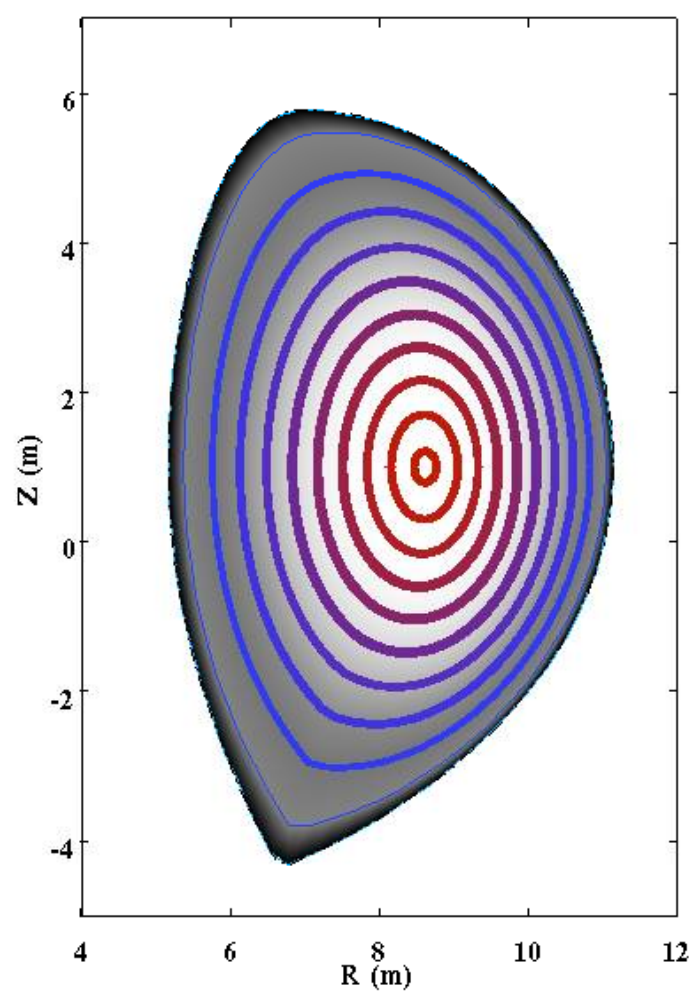

Fig. 10 : DEMO2: METIS magnetic equilibrium in the stationary phase (low radiation case)
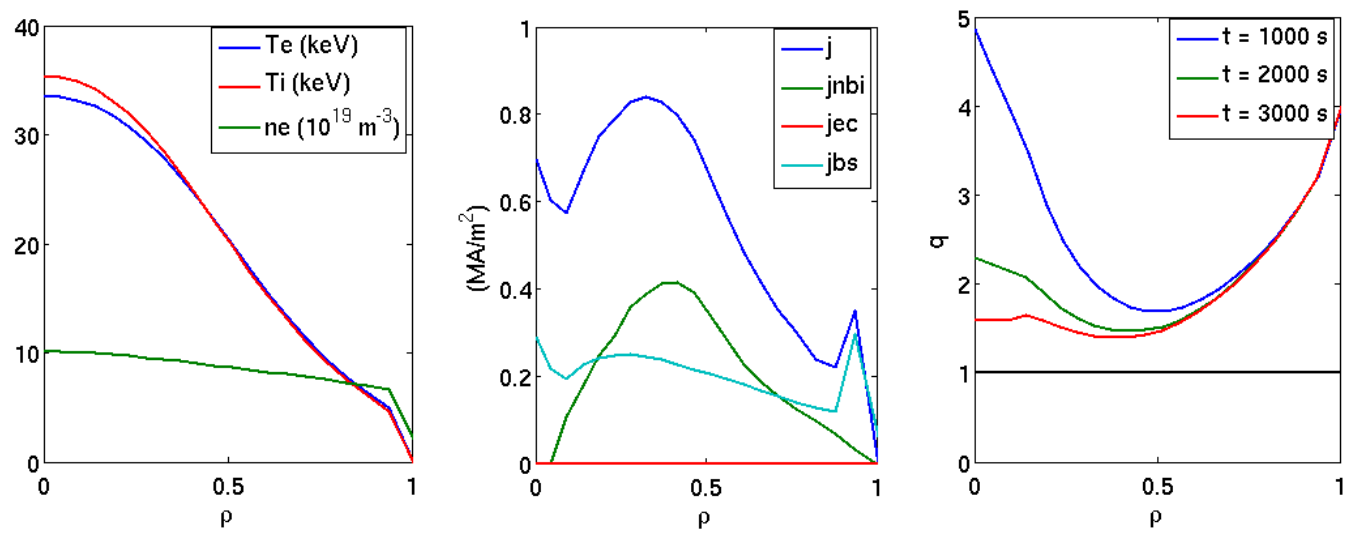

Fig. 11 : DEMO2 METIS simulation (low radiation case). From left to right: 1) radial profiles in the stationary phase of electron and ion temperature, electron density; 2) total current density and NBI, EC, bootstrap current density sources; 3) safety factor profile at three different times. 

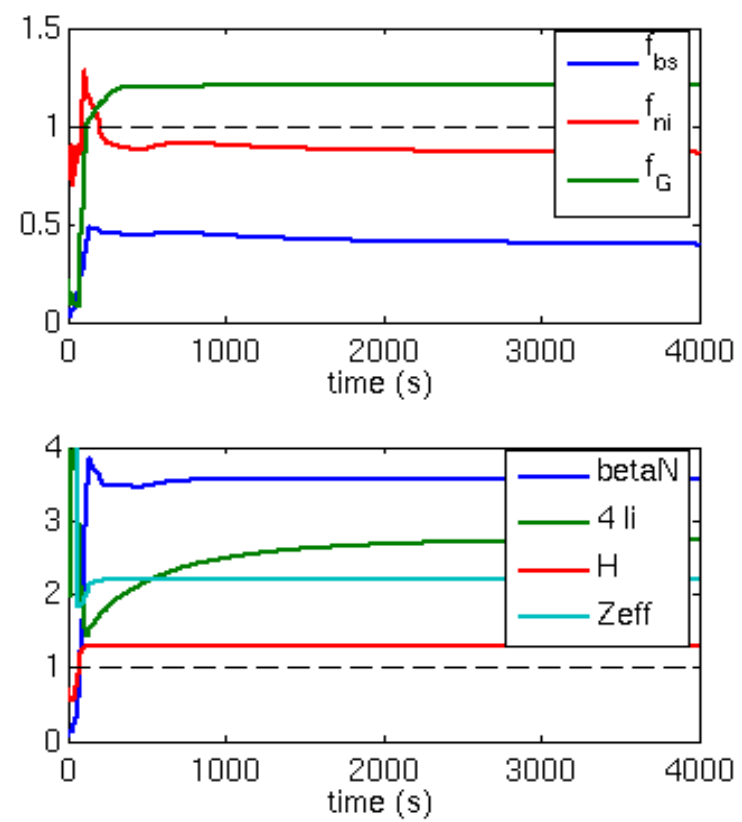

Fig. 12 : DEMO2 METIS simulation (low radiation case). Top: bootstrap, non-inductive current and Greenwald fractions. Bottom: $\beta_{N}, 4 l_{i}, H$ factor and $Z_{\text {eff. }}$.
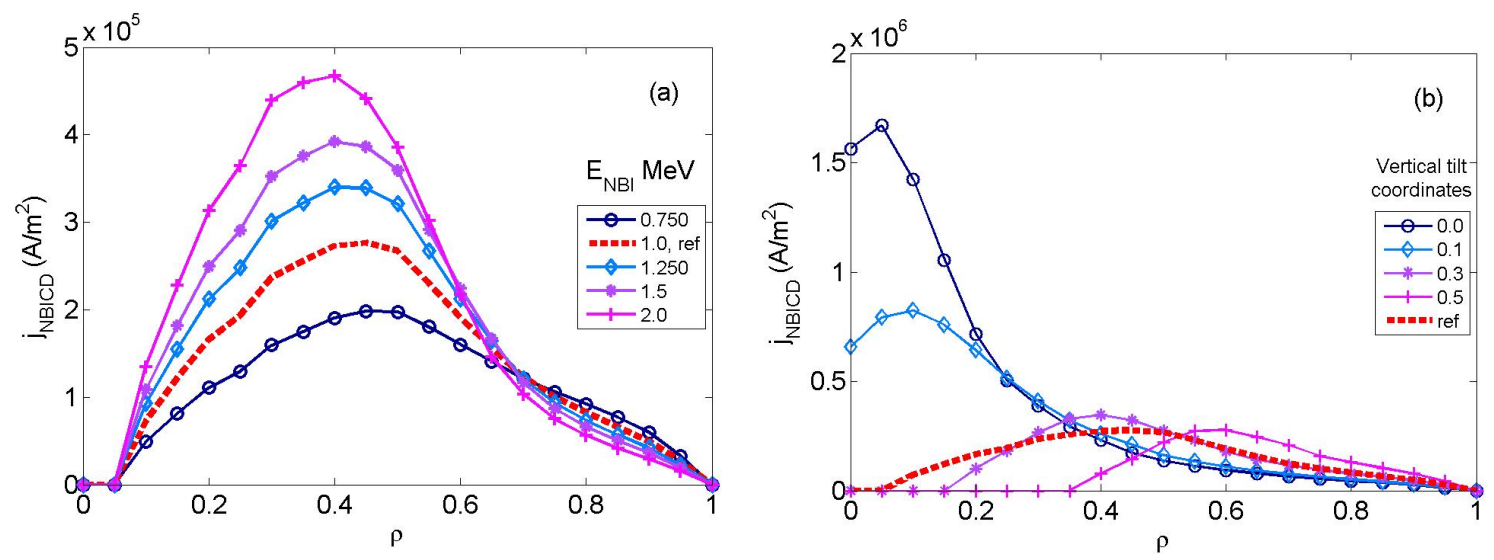

Fig. 13 : Profiles of current density driven by NBI. Figure 13(a) shows a scan on the injection energy, Figure 13(b) a scan on the vertical tilt of the beam. The vertical tilt coordinate is defined as the normalized coordinate [ $0=$ centre, $1=L C F S]$ along the vertical axis where the beam line projection intersects the axis. 


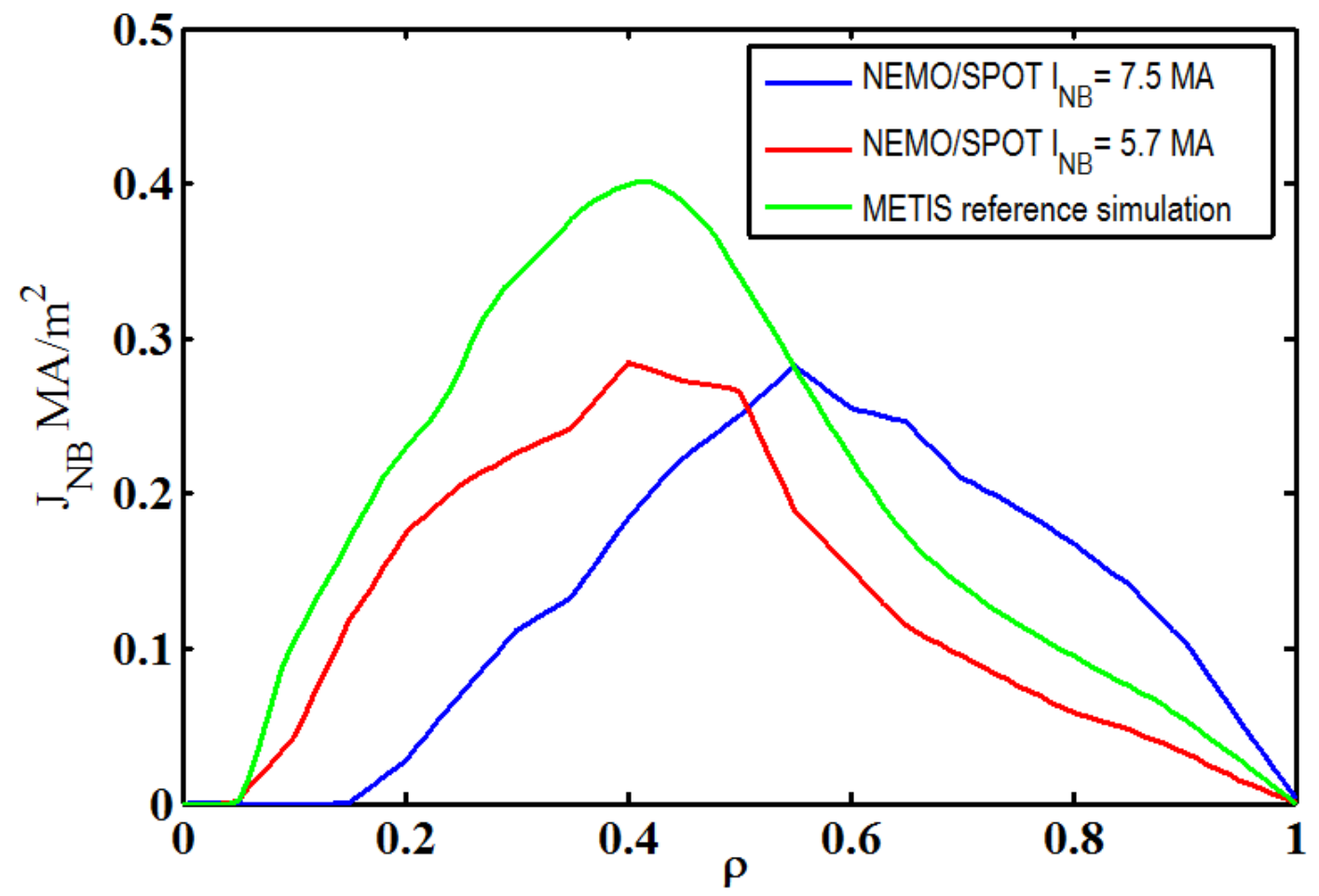

Fig. 14 : Profiles of current density driven by NBI for the DEMO2 working point. METIS simulation (low radiation case), and NEMO/SPOT simulations for two different injection angles.

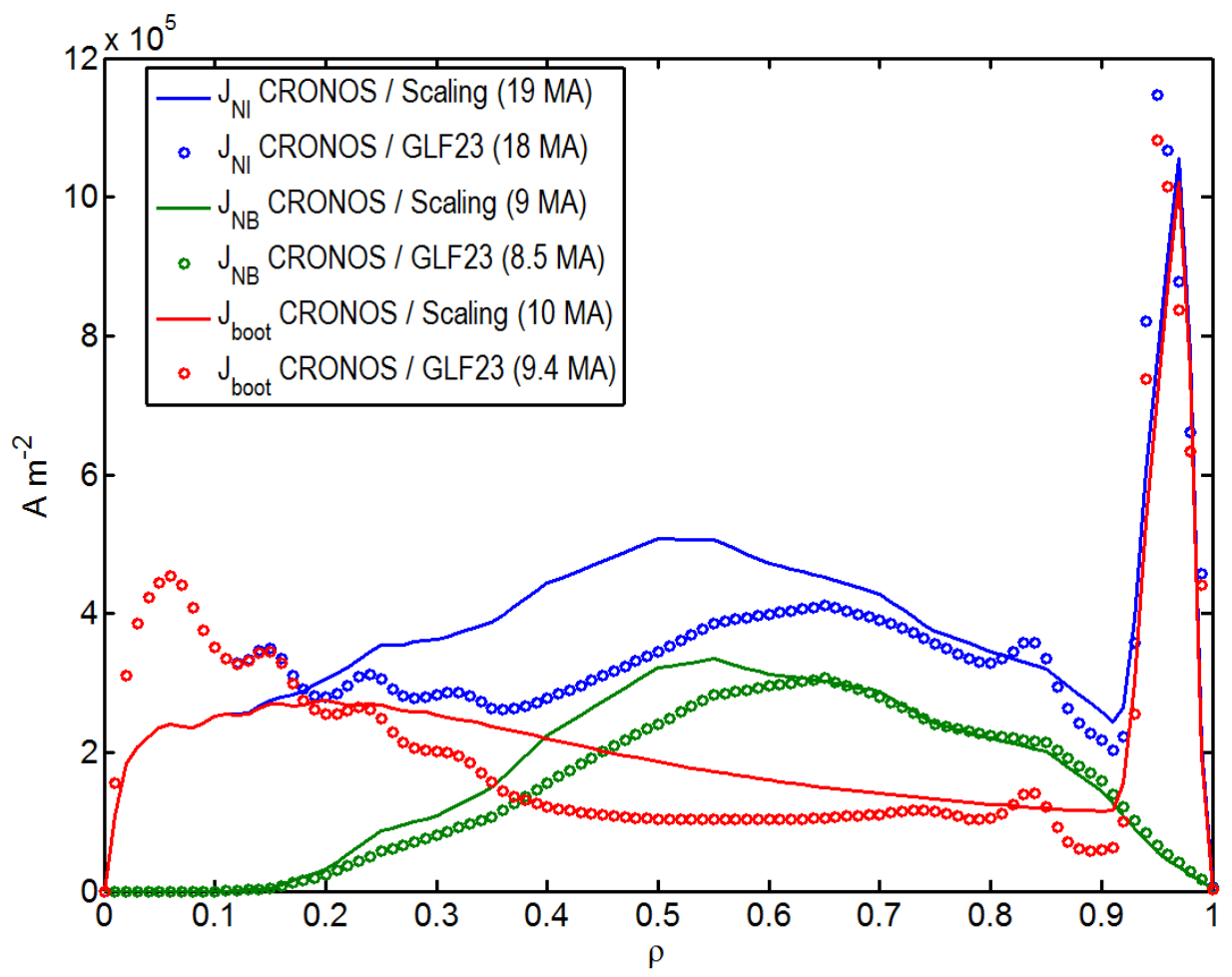

Fig. 15 : Profiles of non-inductive, bootstrap and NBI driven current densities computed by CRONOS for the DEMO2 reference working point. Inside CRONOS, heat diffusivity is renormalised to yield the H-mode scaling (solid lines) or computed from the GLF23 first principle transport model (circles). 


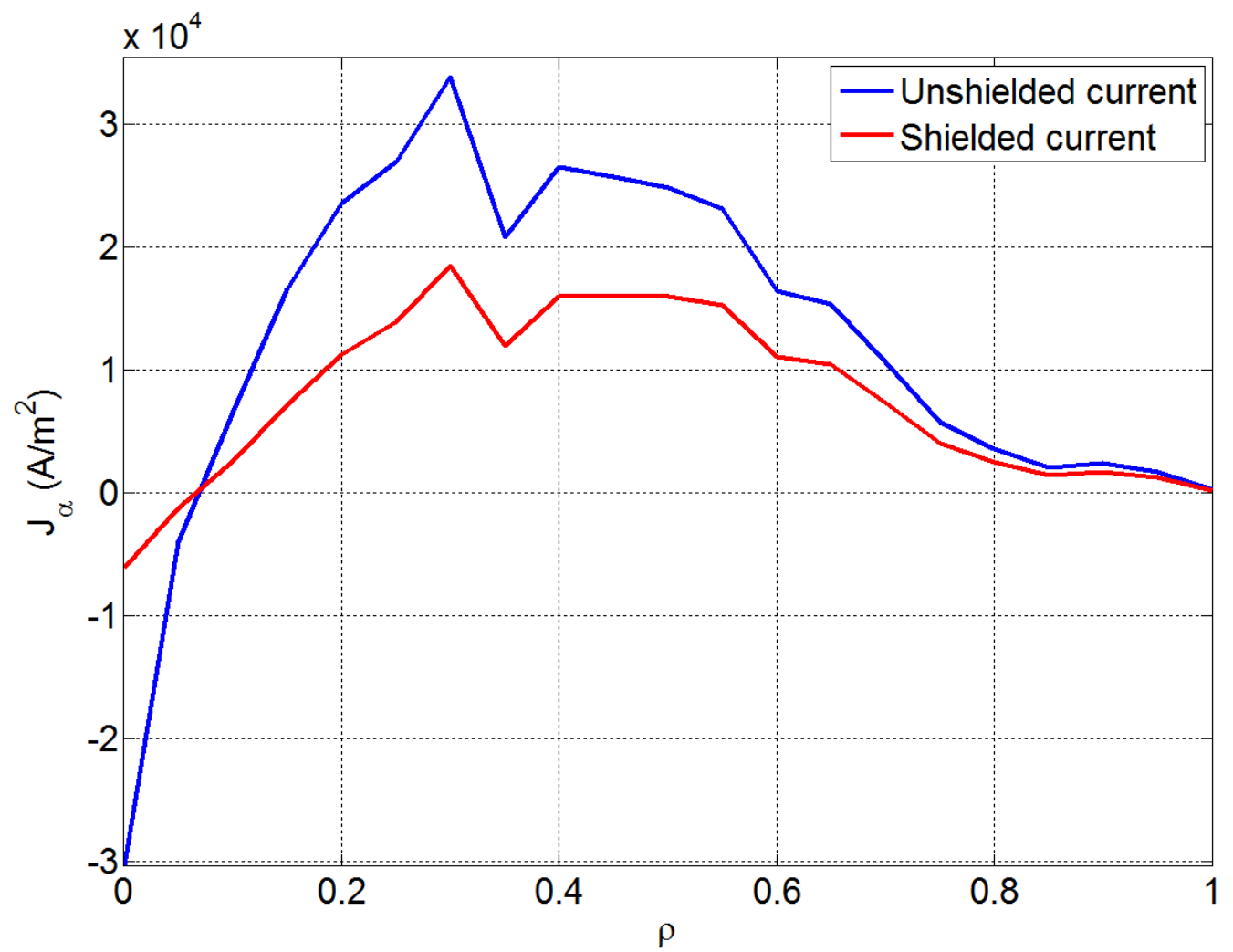

Fig. 16 : Profiles of current density driven by the alpha particles, with and without the electron shielding effect.From CRONOS simulation for the DEMO2 reference working point. The alpha particle distribution is computed by means of SPOT.
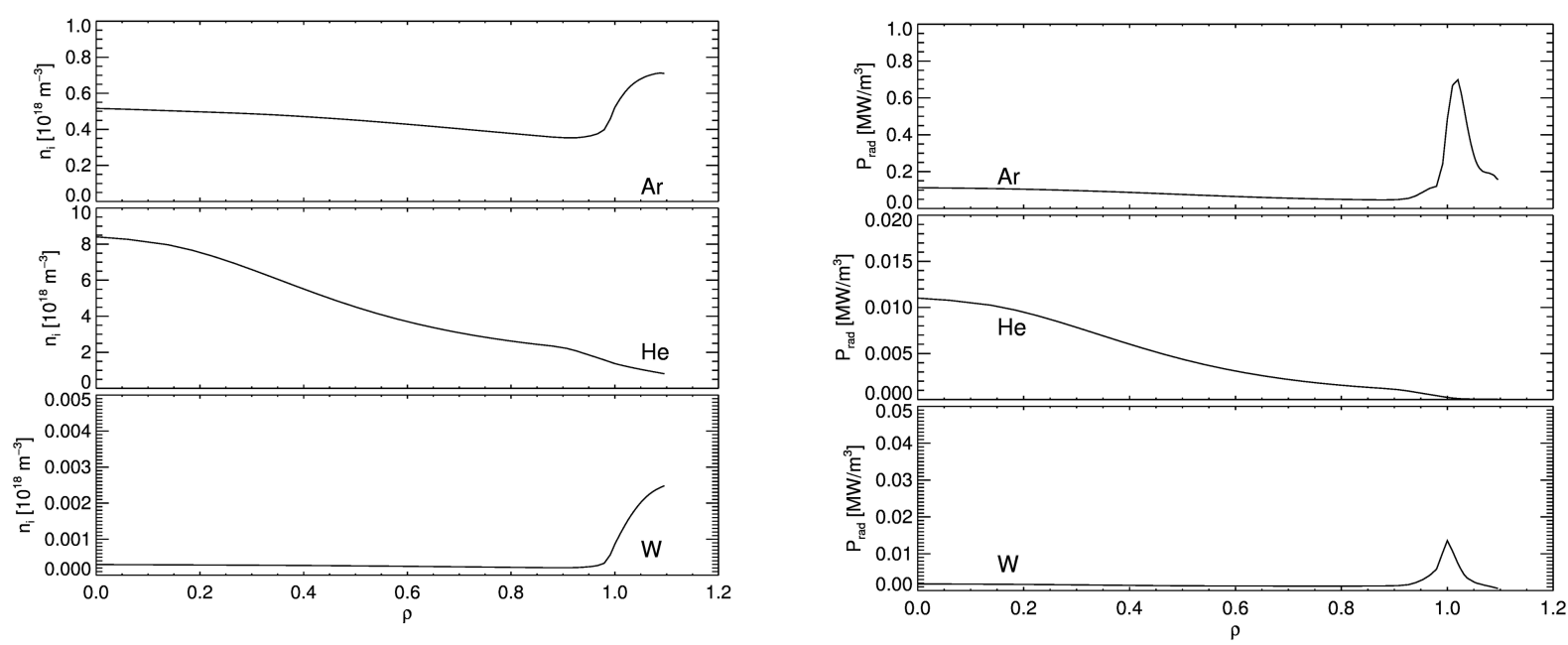

Fig. 17 : Argon, Helium and Tungsten density profiles vs normalized radius in the stationary phase of DEMO2, simulated by JINTRAC, with the SANCO impurity model (a), left; radiated power density profiles by the three impurities $(b)$, right. 